\title{
VI. Delta rays from gases
}

\section{Norman Campbell Sc.D.}

To cite this article: Norman Campbell Sc.D. (1914) VI. Delta rays from gases, Philosophical Magazine Series 6, 27:157, 83-91, DOI: 10.1080/14786440108635064

To link to this article: http://dx.doi.org/10.1080/14786440108635064

$$
\text { 册 Published online: } 08 \text { Apr } 2009 .
$$

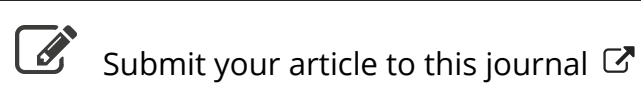

\footnotetext{
Џll Article views: 2
}

Q View related articles $\sqsubset$ 
granite is so much greater than that of basalt. It is just possible that frequent heating and cooling of some specimens of basalt might cause the development of the more minute system of cracks to such an extent as to cause a considerables permanent lowering of the conductivity accompanied by a change in the effect produced by heating, so that the conductivity would fall like that of granite. "This is just what was noticed in the first basalt determinations. but these, as has been shown, were not of a sufficiently reliable nature for much stress to be laid on their results.

It would seem as a result of all the determinations that for temperatures up to $500^{\circ}$ or $600^{\circ} \mathrm{C}$. the conductivity of the earth's crust may be taken as about $4 \times 10^{-3}$ without risk of serious error, unless the conductivity is sensibly al'ected by the large pressures involved.

In conclusion I wish to express my sincere gratitude to Dr. Joly for his kind interest and many valuable suggestions during the progress of the work.

Physical Laboratory,

Trinity College, Dublin,

Oetober 6th, 1913 .

\section{Delta Rays from Grases. By Norman Campbeli, S.L.}

1, T $\mathrm{T}$ is generally believed that when the molecules of a gas are ionized the electrons are expelled from them with velocities similar to those of the $\delta$ rays from metals; the belief is based upon the exact parallelism of the ionizing powers of rays and their power of exciting $\delta$ rays from metals. There appears to be no direct experimental support for the belief, no observations on the ionization of gases which make it necessary to believe that the electrons ejected in ionization have an average initial velocity of several volts $\dagger$. The absence of such observalions appears to be due to the fact that measurements of the ionization of gases have been made at too great pressures or in too great electric fields.

* Communicated by the Author.

+ Kleeman (Proc. Roy. Soc. lxxxiii. p. 195 (1910)) produced evidence that the electrons liberated in ionization possess a finite average momentum in the direction of that of the jonizing rays; but, since it appears that the $\delta$ rays from metals do not possess such a momentum, his experiments, whatever their interpretation, do nut support the belief mentioned. 
If the pressure is too great the electrons ejected in ionization lose their initial velocities by collision with the molecules before they can make their presence felt at the electrodes; if the electric field is too great their initial velocities are inappreciable compared with those that they acquire in moving under the field. Evidence for the existence of $\delta$ rays in gases would be best obtained by studying the ionization of a gas at a very low pressure in a field such that the greatest difference of potential is not great compared with that of the initial velocity of the $\delta$ rays.

2 . Let us consider what should be the relation between $i$, the current through a gas contained in a parallel plate condenser, and $V_{0}$, the potential difference between the plates, when the pressure of the gas is so low that collisions between the electrons liberated and the molecules of the gas are negligibly rare. The current will consist of three parts. (1) There will be the current carried by the $\delta$ rays from the electrodes. If the same number, $\mathrm{N}$, of $\delta$ rays are liberated from each electrode, and if $f(\mathrm{~V})$ is the fraction of these rays emitted in such a direction and with such a velocity that they cannot reach the opposite electrode against an opposing potential $\mathrm{V}$, then this part of the current is $\mathrm{N} e \cdot f^{\prime}\left(\mathrm{V}_{0}\right)$. N and $f\left(\nabla_{0}\right)$ can be determined by measurements when the pressure of the gas is zero *. (2) There will be the current carried by the positive ions which are left when the molecules are ejected. The "initial velocity" of these positive ions, due to the reaction from the electrons ejected, will, if the velocity of those electrons is nearly the same as that of the $\delta$ rays from metals, be less than 0.001 volt and small compared to their velocity of thermal agitation at room temperatures $(0.033$ volt). Since in the circumstances considered recombination will not occur, the number of these ions arriving at the oppositely charged electrode will be determined by their diffusion; this part of the current will be similar to that studied in a recent paper $t$. If $n$ is the number of such ions formed per second this part of the current will be

$$
n e\left(1-2 \mu / \mathrm{V}_{0}\right) \text {, }
$$

where $\mu$ is about 0.025 volt at room temperatures. (3) There will be the current conveyed by the $\delta$ rays from the gas.

* Some of the $\delta$ rays arriving at the electrodes are reflected there (cf. Phil. Mag. Aug. 1911, p. 276 and Jan. 1912, p. 46), but, if we may assume that reflexion affects the $\delta$ rays from gases in the same way as those from metals, the following considerations are independent of the effects of reflexion.

† Phil. Mag. Nov. 1913, p. 912. 
The total number of such rays produced is, of course, again $n$. If $f^{\prime}(\mathrm{V})$ is the fraction of these rays so emitted from the molecules that they cannot reach the negative electrode when the difference of potential between that electrode and the place where they are produced is $\mathrm{V}$, then it is easy to show that this part of the current is

Hence we shall have

$$
\frac{n e}{\mathrm{~V}_{0}} \int_{0}^{\nabla_{0}} f^{\prime \prime}\left(\mathrm{V}_{0}\right) d \mathrm{~V}
$$

$i / e=\mathrm{N} f\left(\mathrm{~V}_{0}\right)+n\left(1-2 \mu / \mathrm{V}_{0}\right)+\frac{n}{\mathrm{~V}_{0}} \int_{0}^{\mathrm{v}_{0}} f^{\prime}(\mathrm{V}) d \mathrm{~V} . . .$.

3. Now let us consider how this equation will be modified when the pressure of the gas is not so low that the effects of collisions between electrons and molecules are wholly negligible. Valuable information as to the results of such collisions is provided by the recent work of Franck and Hertz * They show that, when the gas is hydrogen or helium, the number of collisions made by an electron in travelling through the gas can be determined from the mean free path of an electron calculated from the data of the dynamical theory of gases; and also that, as a result of the collision, the electron is usually reflected with a very small loss of energy. Their experiments have not yet been extended to gases, such as oxygen, which possess a marked "affinity for electrons," but they think it probable that the number of collisions in this case is again calculable from the gas theory free path, while the electron is not reflected but adheres to the molecule with which it collides.

When an electron adheres to a molecule it becomes an ion very similar to the positive ions which carry the part of the current (2). In all the cases which we shall consider this part of the current is very nearly suturated, and hence we may suppose that every electron which adheres to a molecule arrives ultimately at the oppositely charged electrode, whether it would or would not have done so if the collision had not occurred. Assuming that the proportion of electrons which can travel a distance $x$ without collision is $e^{-x / \lambda}$, where $\lambda$ is the free path of an electron and is taken to be independent of its velocity within the limits considered, we find in place of (1)

$$
\begin{aligned}
i / e=\mathrm{N}\left[1-e^{-\frac{l}{\lambda}}\{1\right. & \left.\left.-f\left(\mathrm{~V}_{0}\right)\right\}\right]+n\left(1-2 \mu / \mathrm{V}_{0}\right) \\
& +\frac{n}{\mathrm{~V}_{0}} \int_{0}^{\mathrm{V}_{0}}\left[1-e^{-\lambda \mathrm{V}_{0}}\left\{1-f^{\prime \prime}(\mathrm{V})\right\}\right] d \mathrm{~V} . .
\end{aligned}
$$

* J. Franck \& G. Hertz, Deutsch. Phys. G'es. xv. 9, p. 373 (1913). 
It is to be observed that the effect of the collisions with the molecules of the gas is to increase both the first and the third terms on the right hand of the equation.

When the gas is one which has no affinity for electrons and reflects rather than absorbs them, the effect of collisions is somewhat different. It is now possible that a $\delta$ ray starting from one electrode may be reflectod back to that electrode; the number of such $\delta$ rays crossing the condenser is diminished. Further, since there is little loss of energy at reflexion, and since the reflecting power of the molecules does not change rapidly with the speed of the electrons, the proportion of the electrons starting from one electrode which are deflected back to it will be nearly the same whether they are emitted with or against the field, so long as the potential difference between the electrodes is small compared with the velocity of emission. The collisions with the molecules will diminish the part of the current due to the $\delta$ rays from the electrodes for such small potentials, while in the case of unelastic collision they increased it. On the other hand collisions, even if perfectly reflecting, will not diminish the current carried by the $\delta$ rays from the gas, for these electrons, must arrive ultimately at one of the two electrodes and cannot cease to act as carriers by being reflected to the place where they were generated. The current carried by these $\delta$ rays depends on the ratio of those which arrire ultimately at the oppositely charged electrode to those which arrive at the similarly charged electrode; reflexion cannot decrease this ratio; it will increase it if there is any loss of energy at reflexion. Accordingly the third part of the current will be increased whether the collisions are elastic or unelastic, but to a greater extent in the second case.

The second part of the current is independent of the number of collisions, and in any case it is al ways very nearly saturated.

4. Let us now examine the experimental facts in the light of the foregoing discussion. Some apparently strange results of measurements of the current through ionized air and hydrogen at low pressure were described in a former paper*; they are now set out rather more fully in Tables I. and II. The distance between the electrodes as well as the pressure of the gas was varied ; both quantities are expressed by the ratio $l / \lambda$, where $l$ is the distance between the electrodes and $\lambda$ the calculated free path of an electron in the gas; this ratio is given in the top row. 'The second jow gives the value of $n / \mathrm{N}$. Ne is the saturation current at yero pressure; $n$ cannot be found by direct measurements of the saturation * Phil. Mar. ()et. 191: p. 5.27. 
eurrent at the low pressure, for at these pressures the current is never saturated; ionization by collision occurs before saturation. But if it be assumed that $n$ is proportional to the pressure, its value for low pressures can be determined from observations at high pressures, when saturation is attainable. For the purposes of the tables $n$ is calculated by means of this assumption. The remaining figures in the

TARLE I.-Hydrogen.

\begin{tabular}{|c|c|c|c|c|c|c|c|c|c|c|c|c|}
\hline$l_{i} \lambda \ldots \ldots$ & 0 & $0 \cdot 68$ & $1 \cdot 24$ & 1.98 & $3 \cdot 35$ & 6.32 & $7 \cdot 28$ & $10 \cdot 0$ & $11 \%$ & $17 \cdot 4$ & $26 \cdot 3$ & Cale: \\
\hline$\| / \mathrm{N} \ldots$ & 0 & $0 \cdot 10$ & 0.18 & 0.28 & $0 \cdot 48$ & 0.91 & 0.99 & $1 \cdot 36$ & $1 \cdot 68$ & 250 & $3: 59$ & \\
\hline & $0.46 \overline{5}$ & $\overline{i-0}$ & 0.28 & 0.37 & 0. & 0.65 & $0 \cdot 64$ & 0.74 & 0.79 & 0883 & 0.93 & 11.59 \\
\hline & $0 \cdot 651$ & $(-0.17)$ & 0.09 & 0.28 & 0.42 & 0.62 & 0.60 & 0.71 & 0.76 & 0.82 & 0.93 & (1). 19 \\
\hline 3 & 0.768 & $(-0 \cdot 13$ & 0.16 & 0.25 & $0: 36$ & 0.59 & 0.58 & 0.69 & 074 & 0.81 & 0.91 & 0.7 \\
\hline$\because$ & 0.887 & 0.03 & 0.31 & $0 \cdot 29$ & $0 \cdot 42$ & 0.59 & $0 \div 58$ & 0.68 & 0.74 & 080 & 091 & (1)81 \\
\hline 10. & 0.981 & 0 & $0.5 \overline{5}$ & 0.61 & 0.57 & 0.67 & 0.65 & 0.74 & 078 & 0.83 & 0.93 & (1).89 \\
\hline 20 & 0.997 & 1.08 & 1.26 & $1 \cdot 10$ & 0.95 & 0.87 & 0.83 & 0.95 & 087 & $0: 89$ & 1.01 & $0.9 t$ \\
\hline 30. & 1.000 & 1.7 & $1 \cdot 77$ & 1.63 & $1 \cdot 34$ & $1 \cdot 08$ & 1.04 & 0.99 & 0.96 & 0.93 & 1.07 & 0.05 \\
\hline 50. & - & $2 \cdot 36$ & $2 \cdot 53$ & $2 \cdot 41$ & $2 \cdot 14$ & $1 \cdot 68$ & $1 \cdot 61$ & $1 \cdot 38$ & 1.24 & 1.07 & $1 \cdot 24$ & 0.97 \\
\hline
\end{tabular}

TABLE II.-Air.

\begin{tabular}{|c|c|c|c|c|c|c|c|c|c|c|c|}
\hline$l \lambda \ldots .$. & 0 & 0.35 & $0 \cdot 76$ & $1 \cdot 43$ & $2 \cdot 34$ & $2 \cdot 76$ & $3 \cdot 63$ & 460 & $7 \cdot 75$ & $10^{\circ} 0$ & Cale. \\
\hline$n_{i} \mathbf{N}$. & 0 & $0 \cdot 10$ & 0.24 & $0 \cdot 42$ & 0.71 & 0.82 & $1 \cdot 16$ & 1.39 & $2 \cdot 48$ & $3 \cdot 00$ & \\
\hline$=1$. & 0.465 & $0 \cdot 40$ & 0.60 & 0.62 & 0.57 & 060 & 0.56 & 0.58 & 0.61 & 0.66 & 0.59 \\
\hline 2 & 0.651 & 053 & 0.63 & 0 (i8 & $0 \cdot 65$ & 067 & 065 & 067 & 0.68 & $0 \cdot 70$ & 0.69 \\
\hline 3 & 0.768 & 0.56 & 0.64 & $0 \cdot 68$ & 070 & $(1.67$ & $0 \cdot 69$ & 0.70 & 0.72 & $0 \cdot 71$ & $0 \cdot 74$ \\
\hline 5.. & 0.887 & 0.66 & $0 \cdot 66$ & 0.70 & 0.76 & 0.70 & 0.75 & 0.76 & 0.78 & 0.74 & 0.81 \\
\hline 10. & 0980 & 0.83 & 0.75 & 0.78 & 0.82 & 0.78 & 0.84 & 0.84 & 0.86 & $0 \cdot 81$ & $0 \cdot 89$ \\
\hline 20. & 0.997 & 0.96 & 0.98 & 0.97 & 0.97 & 0.94 & 0.95 & 0.97 & 0.93 & 0.87 & 0.94 \\
\hline 30. & $1 \cdot 000$ & 1.04 & $1 \cdot 19$ & $1 \cdot 16$ & $1 \cdot 18$ & $1 \cdot 15$ & $1 \cdot 12$ & $1 \cdot 14$ & $1 \cdot 01$ & 0.84 & 0.95 \\
\hline 50. & - & $1 \cdot 33$ & 1.55 & 1.52 & $1 \cdot 62$ & 1.59 & 1.55 & 157 & $1 \cdot 33$ & $1 \cdot 19$ & 0.97 \\
\hline
\end{tabular}

first column give the fraction of the total current * at zero pressure carried by the $\delta$ rays from the electrodes which is obtained with the potential difference in the corresponding row of the first column; they also give the values of $f(\mathrm{~V})$

* The o-ray current is taken to be saturated at 30 volts. It has been noted by several observers that at ligher voltages the current decreases, and this decrease has been explained by Bumstead as due to the emission of $\delta$ rays with much higher speeds which cause the emission of the low speed $\delta$ rays. 'The high speed $\delta$ rays are here left out of account, for' though probably much of the innization is really due to them, they are so few in number and have peeds so high compared to the potentials applied that they do not carry any appreciable portion of the current. 
for those potentials. The other columns give the values of $i_{p} \frac{-i_{0}}{n e}$ for the various potentials, where $i_{p}$ is the measured current through the condenser at the pressure concerned, $i_{0}$ that with the same potential at zero pressure. If (1.) were applicable they would thus represent the part of the current due to the gas and would be equal to

$$
1-2 \mu / \mathrm{V}_{0}+\frac{1}{\mathrm{~V}_{0}} \int_{0}^{\mathrm{V}_{0}} f^{\prime}(\mathrm{V}) d \mathrm{~V}
$$

The last column (marked calc.) gives the values of this expression on the assumption that the $\delta$ rays from a layer of gas are emitted with the same speeds as those from the surface of a metal, so that $y^{\prime}(\mathrm{V})=f(\mathrm{~V})$. The integral is calculated by quadrature from the numbers in the first column. The probable error of the numbers in all the columns but the first is about 0.03 . It is so large, firstly because the currents to be measured are small and the numbers recorded are only differences between measured currents; secondly, because the value of $\mathrm{N}$ is somewhat uncertain. As several observers have found, $\mathrm{N}$ depends in some measure on the gas film on the surface of the electrodes and may alter when the pressure is changed.

5. Let us first consider the results for hydrogen.

The measurements when $V_{0}$ is 20 volts or more are clearly affected by ionization by collision and hence have no bearing on the present discussion. For the rest attention is directed to the following features : first, the numbers increase regularly with $l / \lambda$; second, the numbers in each column do not increase regularly with $V_{0}$ but have a minimum at about 3 volts ; third, the numbers for small valnes of $l / \lambda$ and $V_{0}$ are very much less than those calculated from (1); they are often less than those corresponding to the second term in that equation which represents the current carried by the positive ions only; fourth, in the first columns there are negative numbers, that is to say, the presence of a little gas in the condenser actually diminishes the current flowing through it with small voltages*.

It is clear that none of these features can be explained on the basis of (1); but they may be explained, at any rate partially, on the basis of the discussion of the effect of a small amount of hydrogen. We saw that the main effect of

* The decrease is very small and on the margin of experimental emor, but the experiment was repeated several times and always gave a decrease. 
a little hydrogen was likely to be a decrease in the current carried by the $\delta$ rays from the electrodes. The numbers given in the Table are obtained by subtracting from the measured current that carried by those rays in a vacuum; if the rays carry less current when gas is present, the numbers in the Table will all be smaller than than they would be if (1) were applicable. If, owing to the presence of gas, the current due to the $\delta$ rays from the electrodes is decreased by more than the extra current due to the ionization of the gas, the negative figures in the first column would be explained. The minimum in any column at about 3 volts could be explained if the effect of the field in counteracting the effects of reflexion do not begin to be appreciable until $V_{0}$ is greater than 3. Again, of course, on this view the observed numbers will all be less than those calculated, until the current carried by the $\delta$ rays from the electrodes lecomes inappreciable compared with that due to the gas. But when this stage is reached the effect of collisions in increasing the current due to the $\delta$ rays from the gas is important, so that we immediately pass to the stage where the observed numbers are greater than the calculated. The data concerning the reflexion of the electrons by collision are not so definite that any attempt can be made to explain the results quantitatively; and certainly it seems impossible to deduce from these results any conclusion concerning the initial velocity of the $\delta$ rays from the gas.

6. Now let us turn to the results for air. Here again, the measurements when $V_{0}$ is 30 volts or more are affected by ionization by collision. Again, also, there is a general increase in the numbers in each row with $l / \lambda$, but the increase is not nearly so marked ar before; the numbers are approximately constant between $l / \lambda=1$ and $l / \lambda=4$, and in this range they show some approximation to those calculated from (1). The numbers in each column increase regularly with $V_{0}$, but the rate of increase is small in the region where in the previous case there was a minimum.

In interpreting these results we must remember that, of the two gases in air, nitrogen has no affinity for electrons * and probably acts like hydrogen, while oxygen has a strong. affinity. Accordingly the nitrogen will tend to decrease the current carried by the $\delta$ rays from the electrodes, and so make the numbers smaller than those predicted by (1), while the oxygen tends to increase both this current and that due to the $\delta$ rays from the gas, and so make the numbers too

* See the measurements on the velocities of the ions by Franck, Deutsch. Phy*. Gesell. xii. p. 613 (1910). 
large. It is possible, accordingly, that the smallness of the numbers for $l / \lambda=0.35$ is due to the preponderant action of the nitrogen, while the constancy of the numbers for somewhat greater values of $l / \lambda$ is due to the compensating action of the two gases. At any rate, the difference between the results for hydrogen and air, the fact that the numbers are almost uniformly greater in the case of air for the same value of $\nabla_{0}$ and $l / \lambda$, is exactly what would he expected to be the result of the prescnce of a strongly electronegative gas.

But the main interest of the experiments lies in the question whether they can give any information as to the initial velocity of the $\delta$ rays from gases. It appears necessary to suppose that these $\delta$ rays have a finite initial velocity, and that it is of the same order of magnitude as that of the $\delta$ rays from metals. No other explanation can, I think, be given of the fact that in no case is the current through the gas saturated when $V_{0}=20$, even though the currents due to the $\delta$ rays from the electrodes and that due to the positive ions is saturated with that potential. If the constancy of the numbers in the Table over a certain range of $l / \lambda$ means that within this range (1) is applicable, we shoula have to conclude that the $\delta$ rays from the gas are, on the average, a little faster than those from the electrodes, since the numbers in the Table are a little smaller than those calculated on the assumption that $f(\mathrm{~V})=f^{4}(\mathrm{~V})$.

However, it does not appear that this method is capalule of giving any very precise indications concerning the properties of these rays. They do appear to show that it is necessary to suppose that the rays have a finite initial velocity, and so provide evidence for an important proposition which was universally accepted but had never been directly proved. But this discussion has been published mainly becanse a brief account of the measurements had been given without any attempt at an explanation of them. It seems that they are generally in accordance with the recent addition to our linowledge of the interaction between electrons and molecules.

\section{Summary.}

The theory is considered of the conduction through an ionized gas when the pressure of the gas is so low that the free path of an electron is not small compared with the distance between the electrodes, and the number of ions made in the gas is not large compared with the number of $\delta$ rays made at the electrodes. The discussion is based 
mainly on the recent work of Franck and Hertz on the collisions between electrons and atoms.

The results of some measurements published previously are considered in the light of the theory. They seem in general accordance with that work. They cannot be explained quantitatively at present, but it appears that they make it necessary to believe that $\delta$ rays are emitted from ionized molecules of gases with velocities of the same order of magnitude as those from metals.

Leeds University, August 1913.

VII. An Experiment on Rotatory Polarizution in Liquids. By E. Talbot Paris and Alfred W. Porter, F.R.S.*

T $\mathrm{HE}$ experiment recorded here was carried out in order 1 to see if any evidence could be obtained of a directive action on the molecules of an optically active substance in solution by light passing through the solution. If such a directive action existed (the light tending to orientate the molecules in a certain direction relative to its direction of propagation), it would be expected that when light first passes into the liquid a small interval of time would be required for the full rotatory power to be developed. In this case, if an intermittent source of light were used, giving flashes of sufficiently short duration, the rotation of the plane of polarization would be less than that for a steady source. In the experiment described below the rotation produced in a long tube of cane-sugar solution was measured, first using a steady source of light (a mercury lamp), and afterwards an intermittent one in the form of a platinummercury spark.

The arrangement of the apparatus is shown in fig. 1.

Light from the source $S$ (the mercury are or spark as the case may be) passes through the collimating lens $L_{1}$ and the polarizing nicol $\mathrm{N}_{1}$. Sensitiveness is obtained by means of a half-wave plate of mica at $M$, placed across the middle of the field so as to give a "three-field" arrangement. The sensitiveness can be altered by rotating $\mathrm{N}_{1}$. The sugar solution was contained in a long iron tube $A B$, fitted with plate-glass ends. To prevent the solution becoming contaminated with the iron, this tube was galvanized; the inside was painted with photographic black to avoid reflexions. The length of the solution in the tube was about $165 \mathrm{~cm}$, and the rotation for the green mercury line

* Communicated by the Authors. 